\title{
Asymptotic equivalence of some adaptive biquad notch filters
}

\author{
V V KRISHNA ${ }^{1}$ and C G HIREMATH \\ Signion Systems Pvt. Ltd., 6-3-569/1/2, Rockdale Compound, Somajiguda, \\ Hyderabad 500 082, India \\ ${ }^{1}$ Present address: ZSP Corporations, 2855, Kifer Road, Suite 200, Santa Clara, \\ CA, 95051, USA \\ e-mail: vvkris@zsp.com; signion@hotmail.com
}

\begin{abstract}
A diverse choice of biquad designs is available for adaptive notch filtering or line enhancement (ANF/ALE) applications. We consider one such biquad proposed over a decade ago by David and coworkers and show its equivalence to two other formulations in terms of their pole-zero pair locations in the $z$-plane. By equivalence, we imply that all these adaptive IIR filters possess comparable asymptotic performance. Further, a simple modification, involving only a normalizing factor, is seen to enhance the performance of this filter. This modified ANF/ALE design is then shown to be equivalent to two other recently proposed adaptive biquads.
\end{abstract}

Keywords. Adaptive IIR filters; adaptive line enhancement; adaptive signal detection; adaptive signal processing; IIR digital filters; poles and zeros.

\section{Introduction}

Adaptive IIR notch filters (ANFs) provide superior performance at a lower computational cost relative to their FIR counterparts in suppressing tonal interference in wideband signals. This advantage holds equally well for adaptive line enhancers (ALEs) as well, where a sinusoid immersed in white noise needs to be extracted. As demonstrated in figure 1 , the twin tasks of notch filtering and line enhancement may be viewed as complementary operations using a common framework. Further details of the topic can be had from overviews by Regalia (1995) and others Krishna \& Hiremath (1995).

As in many other evolving fields, here also we have had considerable reinvention by independent researchers leading to many apparently disparate ANF/ALE solutions. As a result, a melange of adaptive IIR structures is now available. In this article, we look at a few biquad designs for notching or enhancing a single sinusoid in noise (more sinusoids can be handled by a suitable arrangement of multiple biquads) and demonstrate their equivalence so as to reduce the attendant confusion by some extent. By equivalence, we imply that 


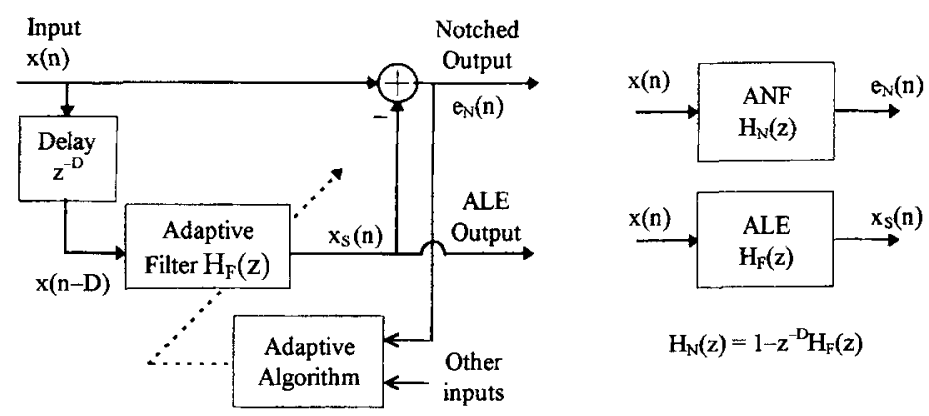

Figure 1. Adaptive notch filter (ANF) block diagram. The structure can be viewed either as an ANF or as an adaptive line enhancer (ALE), depending on the actual output selected. For sinusoids in white noise, a delay $D=1$ is sufficient.

different IIR adaptive filters have identical pole-zero pair locations in the $z$-plane so as to yield comparable asymptotic (infinite data) performance. However, the convergence and tracking properties can differ depending on the specific parameter being updated for adaptation as well as on the adaptation algorithm employed.

As a reference design, we consider the biquad proposed by David and coworkers (David et al 1983; Hush et al 1986), hereafter referred to as the DEESHA filter (using initials of all members in the team). This design is discussed briefly in the following section. We shall then show its equivalence with other filters developed by Cho et al (1990) and Mukund \& Martin (1991). In the fourth section, we consider a modified DEESHA structure for improved performance and examine its relation to two other biquads proposed by Kwan \& Martin (1989) and Regalia (1990).

\section{2. “DEESHA" ANF/ALE}

The DEESHA notch filter is given by

$$
H_{N, 1}(z)=\left[1-\left(\frac{2}{1+r^{2}}\right) \rho z^{-1}+z^{-2}\right] /\left[1-\rho z^{-1}+r^{2} z^{-2}\right],
$$

where $0 \ll r<1$, and $-2 r<\rho<2 r$. The factor $\rho=\left(1+r^{2}\right) \cos \left(\omega_{N}\right)$ is adapted to acquire the notch frequency $\omega_{N}$ while " $r$ " controls the notch bandwidth by appropriate positioning of poles inside the unit circle. The bounds on $\rho$ given above are not tight and may be violated to handle very low (near d.c.) or very high frequency (relative to the sampling rate) sinusoids without any penalty (Krishna \& Hiremath 1995). A simpler form of DEESHA filter suggested by Farhang-Boroujeny (1994) is

$$
H_{N, 1}(z)=\frac{1-2 \rho_{0} z^{-1}+z^{-2}}{1-\left(1+r_{0}\right) \rho_{0} z^{-1}+r_{0} z^{-2}},
$$

where $\rho_{0}=\rho /\left(1+r^{2}\right)=\cos \left(\omega_{N}\right)$ and $r_{0}=r^{2}$. For a fixed $r_{0}$, DEESHA ANF is a constant bandwidth notch filter. That is, its bandwidth is independent of the notch frequency for a sinusoid in white noise. As a result, $\omega_{N}$ converges to the unknown frequency $\omega_{s}$ of the sinusoid without any bias (Krishna \& Hiremath 1995). The notch filter bandwidth reduces 


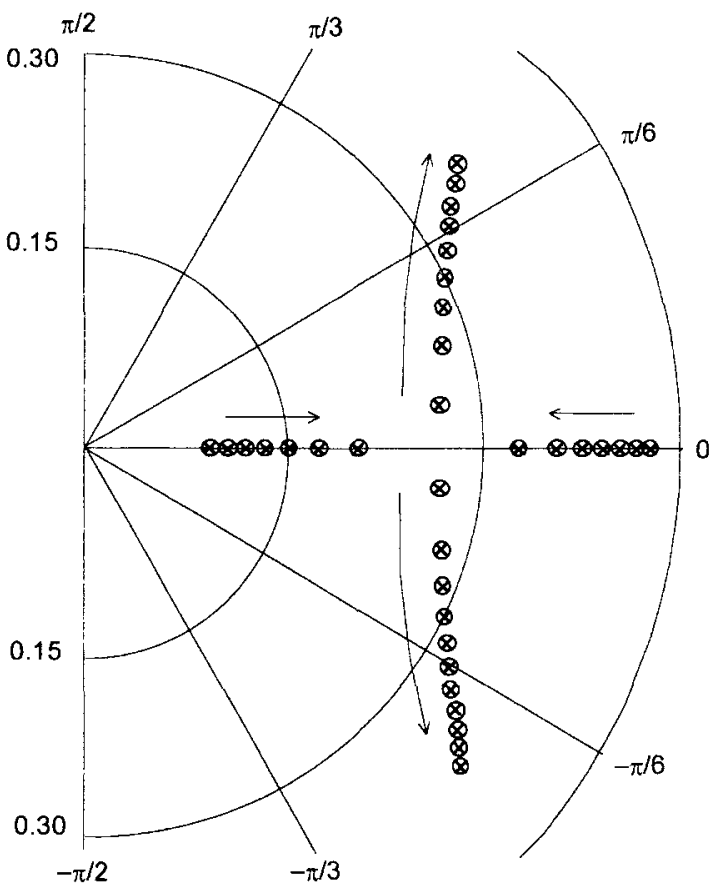

Figure 2. The dependence of pole location on $r_{0}$ for DEESHA ANF. The notch frequency is at $\omega_{N}=\pi / 4$ and the arrows indicate increasing $r_{0}$.

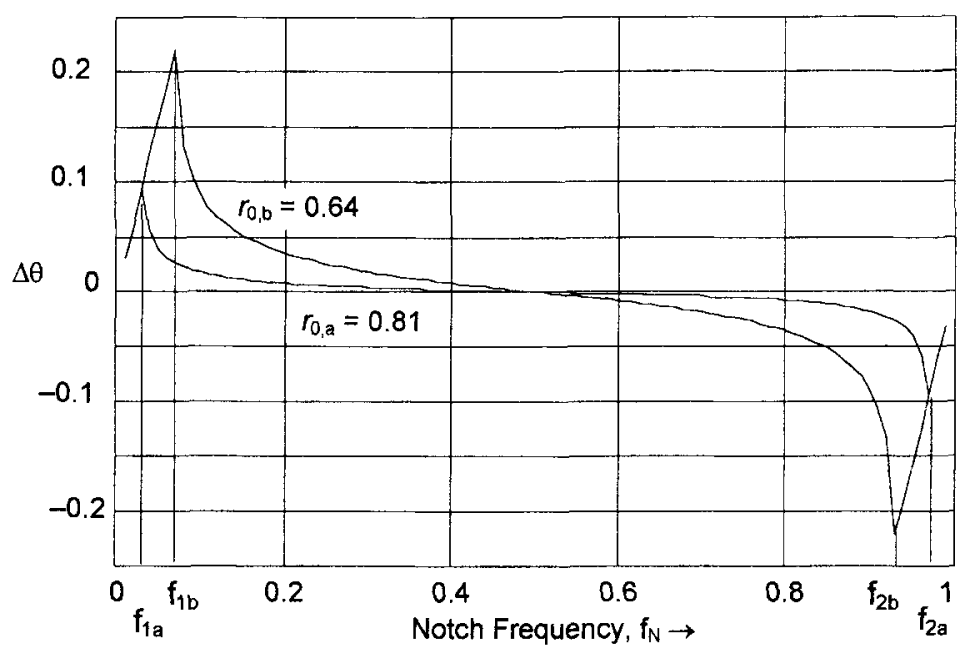

Figure 3. Angular deviation $\Delta \theta$ (in radians), of poles from zeros in DEESHA notch filter. Outside the range $\left(f_{1 x}, f_{2 x}\right)$ where the angular deviation varies linearly, the poles lie on the real axis. 
as a larger value of $r_{0}$ is selected within its upper limit, but at the same time, the adaptive filter convergence and tracking rates will also decrease.

The notch filter requires its zeros to be located on the unit circle at an angle corresponding to the frequency of input sinusoid. The position of its poles is however determined both by the notch frequency as well as by the choice of $r_{0}$. Figure 2 shows some pole positions as $r_{0}$ is varied for notching at frequency 0.25 (normalized). In figure 3 , the angular deviation between a zero and its neighbouring pole is illustrated. Except for the case of a sinusoid with a normalized frequency 0.5 , the poles lie at an angle closer to the real axis than the zeros. At very low or very high frequencies (depending on $r_{0}$ ), the poles may even lie on the real axis itself. This characteristic ensures that the biquad provides a constant bandwidth notch irrespective of the input sinusoid frequency and thereby, a bias-free convergence in the presence of background white "noise".

The band pass filter (BPF) $H_{F, I}(z)$ of the ALE corresponding to DEESHA notch filter $H_{N, 1}(z)$ is given by

$$
H_{N, 1}(z)=\frac{\left(1-r_{0}\right)\left(\rho_{0}-z^{-1}\right)}{1-\left(1+r_{0}\right) \rho_{0} z^{-1}+r_{0} z^{-2}} .
$$

Note that the ANF and ALE are related by $H_{N, 1}(z)=1-z^{-1} H_{F, 1}(z)$. The ALE has its poles at the same location inside the unit circle as ANF, but has only a single real zero lying outside the unit circle. The zero can lie on the unit circle itself, if $\omega_{N}=0$ or $\pi$. The SNR enhancement factor for DEESHA ALE is given by $\left(1+r_{0}\right) /\left(1-r_{0}\right)$.

Figure 4 illustrates the magnitude response of the notch filter and line enhancer based on DEESHA biquad for some values of $r_{0}$.

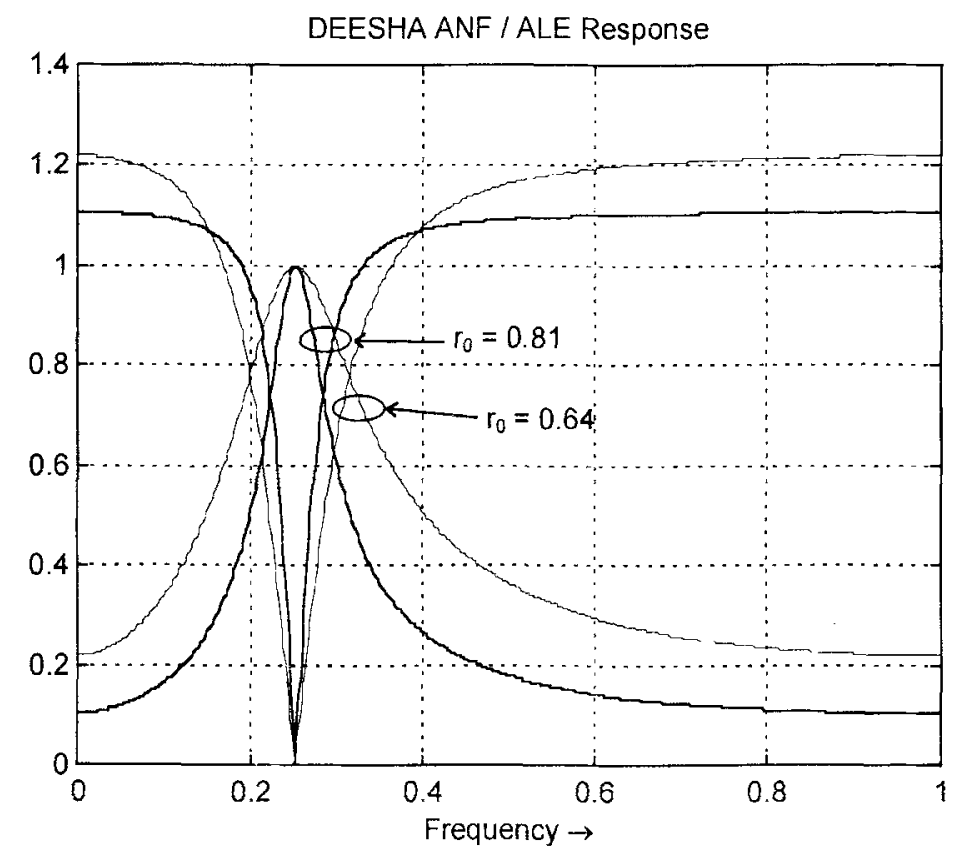

Figure 4. Magnitude response of DEESHA ANF/ALE. The notch is at $f_{N}=0.25$, and $r_{0}=0.81$ and 0.64 . 


\section{DEESHA filter's relatives}

We shall now examine the relation between DEESHA and Cho-Choi-Lee notch filters (Cho et al 1990). This latter design was derived from Rao-Kung filter (Rao \& Kung 1984) using simple approximations and its performance was demonstrated (Cho \& Lee 1993) to be superior to the original Rao-Kung design in terms of reduced frequency bias. The Rao--Kung ANF is given by

$$
H_{N .2}(z)=\frac{1+a_{1} z^{-1}+a_{2} z^{-2}}{1+\alpha a_{1} z^{-1}+\alpha^{2} a_{2} z^{-2}},
$$

where $-2<a_{1}<+2$ and $0<a_{2}<1$ control the notch frequency $\omega_{N}=\cos ^{-1}\left(-a_{1} /\right.$ $\left(2 \sqrt{a_{2}}\right)$ ), while $\alpha$ called pole contraction factor, places poles inside the unit circle on $a$ radial line below the zeros for all notch frequencies. Note that the zeros will lie on unit circle only for $a_{2}=1$, when this ANF becomes identical to yet another notch filter proposed by Nehorai (1985). Unlike the DEESHA filter, both these ANFs are not constant bandwidth filters (i.e. bandwidth now depends on notch frequency) and thus suffer convergence bias induced by white noise component of the input (Krishna \& Hiremath 1995; Regalia 1995). Bias can be a debilitating factor in applications such as phase jitter cancellation in carrier recovery phase lock loops (PLLs) used in high speed modems (Cupo \& Gittin 1989). Cho et al (1990) considered the lattice equivalent of (4) given as

$$
H_{N, 2}(z)=\frac{1+k_{0}\left(1+k_{1}\right) z^{-1}+k_{1} z^{-2}}{1+q_{0}\left(1+q_{1}\right) z^{-1}+q_{1} z^{-2}},
$$

where $q_{0}\left(1+q_{1}\right)=\alpha k_{0}\left(1+k_{1}\right), q_{1}=\alpha^{2} k_{1}, k_{0}=a_{1} /\left(1+a_{2}\right)$ and $k_{1}=a_{2}$. Then, assuming a pole contraction factor $a \approx 1.0$, they used the approximations $q_{0}\left(1+q_{1}\right) \approx k_{0}\left(1+\alpha k_{1}\right)$ and $q_{1} \approx \alpha k_{1}$, and substituted $q_{0}=k_{0}$ and $q_{1}=\alpha k_{1}$ in (5) to obtain

$$
H_{N .3}(z)=\frac{1+k_{0}\left(1+k_{1}\right) z^{-1}+k_{1} z^{-2}}{1+k_{0}\left(1+\alpha k_{1}\right) z^{-1}+\alpha k_{1} z^{-2}} .
$$

For $k_{1}=1$, (which leads to zeros on the unit circle as does the Nehorai filter), this system function finally simplifies to the Cho-Choi-Lee notch filter,

$$
H_{N .4}(z)=\frac{1+2 k_{0} z^{-1}+z^{-2}}{1+k_{0}(1+\alpha) z^{-1}+\alpha z^{-2}} .
$$

where $0<\alpha<1$, and $-1 \leq k_{0} \leq 1$.

Cho \& Lee (1993) termed the design in (7) as a "lattice ANF" and compared its performance with the Rao-Kung and Nehorai filters which were designated as "direct form". This loose choice of terminology creates an impression that the Cho-Lee filter performs better (in terms of bias) somehow because it is a "lattice ANF". Comparing (7) with the DEESHA system function given by Farhang-Boroujeny in (2) earlier, it is immediately obvious that this lattice ANF is simply a reformulation of David et al's design. As such, it has the same pole-zero relationship and bias-free performance edge as does the DEESHA filter over Rao-Kung and Nehorai ANFs. Also, a line enhancer obtained using this "lattice" ANF can be related to the DEESHA ALE in (3) and offers identical SNR improvement ratio.

We now consider an interesting design proposed by Mukund \& Martin. It involves (figure 5) a perfect resonator with poles on the unit circle which is inherently unstable 


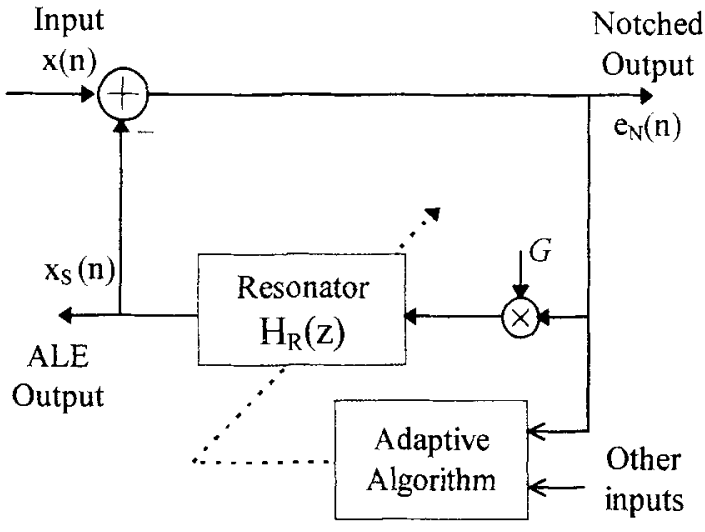

Figure 5. ANF/ALE based on MukundMartin's Resonator in a loop (RIL) structure.

inside a stable feedback loop having a loop gain (or, damping factor) ' $G$ '. Mukund \& Martin explored its use mainly for line enhancement and for estimating the unknown frequency $\omega_{S}$ of a sinusoid but it can be used for notch filtering as well. The resonator, which is a BPF with infinite $Q$-factor is given by

$$
H_{R}(z)=\frac{z_{R}}{z-z_{R}}+\frac{z_{R}^{*}}{z-z_{R}^{*}}=\frac{2 a_{R} z^{-1}-2 z^{-2}}{1-2 a_{R} z^{-1}+z^{-2}},
$$

where $z_{R}=\exp \left(j \omega_{R}\right)$ or $a_{R}=\cos \left(\omega_{R}\right)$ determines the resonant frequency $\omega_{R}$. The filter parameter $a_{R}$ is adapted (Mukund \& Martin 1991) to obtain $\omega_{R}=\omega_{S}$ upon convergence.

To ensure loop stability, we require $0<G<1 / 2$. Using straightforward algebra we can derive the system function between the input $x(n)$ and notched output $e_{N}(n)$ as

$$
H_{N .5}(z)=\frac{1-2 a_{R} z^{-1}+z^{-2}}{1-2(1-G) a_{R} z^{-1}+(1-2 G) z^{-2}} .
$$

Comparing with (2), this is identical to DEESHA ANF for $G=\left(1-r_{0}\right) / 2$ and $a_{R}=\rho_{0}$ ! In fact, the performance of Mukund \& Martin's "resonator in a loop" design depends on the selectable value of loop gain, ' $G$ ', to the same extent as DEESHA ANF depends on $r_{0}$. Note, however that the notch filter bandwidth reduces as $G$ approaches zero.

Similarly, we can also see that the input $x(n)$ and resonator output $x_{S}(n)$ are related by a system function, $z^{-1} H_{F .5}(z)$ where $H_{F .5}(z)$ is identical to the BPF of DEESHA ALE given in (3). This equivalence between DEESHA filter and the more recent MukundMartin's (1993) design may appear surprising or even disappointing, if it were to be naively expected that the infinite- $Q$ resonator in the loop would offer arbitrarily high frequency selectivity. Mukund \& Martin (1991) further showed that their ALE provides an SNR improvement factor of $(1-G) / G$, which exactly corresponds to $\left(1+r_{0}\right) /\left(1-r_{0}\right)$ given by DEESHA.

\section{Enhanced "DEESHA" ANF / ALE}

For moderate values of $r_{0}$ (usually required during initial convergence or tracking time varying frequencies), the DEESHA filter performance is not satisfactory. The SNR improvement 
factor for ALE $\left(1+r_{0}\right) /\left(1-r_{0}\right)$, remains small. Also, the magnitude response of the ANF away from its notch frequency exceeds unity (figure 4). In fact, the response at d.c. and at normalized folding frequency, $f=1$, equals $2 /\left(1+r_{0}\right)$. Both these problems can be simply solved using the structure shown in figure 6 . Here, the new notch filter output $e_{N E}(n)$ is only a scaled version of the original output (the notch filter pole-zero locations in the $z$-plane remain undisturbed). The scale factor ' $S$ ' is selected such that the magnitude response at $f=0$ or 1 , equals unity. That is, we require $S=\left(1+r_{0}\right) / 2$. The modified magnitude response is plotted (figure 7) for two different values of $r_{0}$ and may be compared with the earlier responses.

The scaled output $e_{N E}(n)$ of the notch filter is then used to derive an enhanced ALE signal $x_{S E}(n)$. The enhanced ALE system function $H_{F E}(z)$ given by

$$
H_{F E}(z)=1-H_{N E}(z)=\frac{\left(1-r_{0}\right)}{2} \frac{1-z^{-2}}{1-\left(1-r_{0}\right) \rho_{0} z^{-1}+r_{0} z^{-2}}
$$

has its poles at the same location as $H_{F}(z)$ in (3), but has two real zeros, that remain fixed at $z=+1$ and $z=-1$ on the unit circle. In particular, these fixed zeros are of great help when $r_{0}$ is small (i.e. the filter bandwidth is large). This is brought out in figure 7 where the magnitude response of the enhanced DEESHA filter is plotted. It can be easily shown that the SNR enhancement obtained using (10) equals $2 /\left(1-r_{0}\right)$. Depending on the value of $r_{0}$ selected, the improvement over DEESHA ALE can range from 1 to $2 \mathrm{~dB}$ or more.

We shall now consider two more ANF/ALE designs and draw a relationship with the enhanced DEESHA filter. Both these designs also satisfy the relation $H_{F}(z)=1-H_{N}(z)$ between their BPF and notch filter as in (10) above. That is, there is no explicit delay ahead of the BPF here, unlike the general framework of figure 1 (the "missing" delay, which is essential, is actually incorporated within the notch filter transfer function). Finally, the ALE components of these two designs offer an SNR improvement factor identical to that of enhanced DEESHA.

The first of the two designs was proposed by Kwan \& Martin (1989). To conserve space, we look only at the BPF (in ALE) here, as the corresponding notch filter can be easily

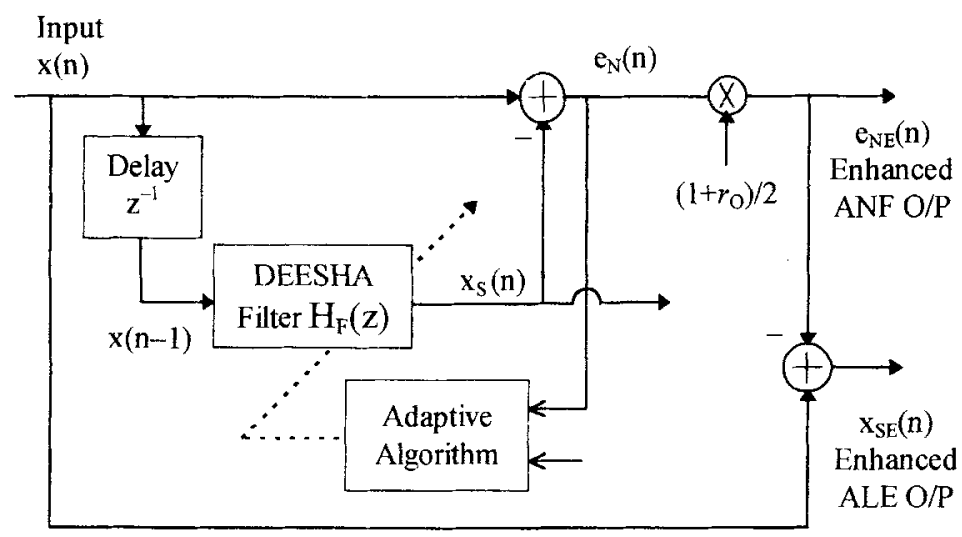

Figure 6. Modification to enhance DEESHA ANF/ALE performance. 


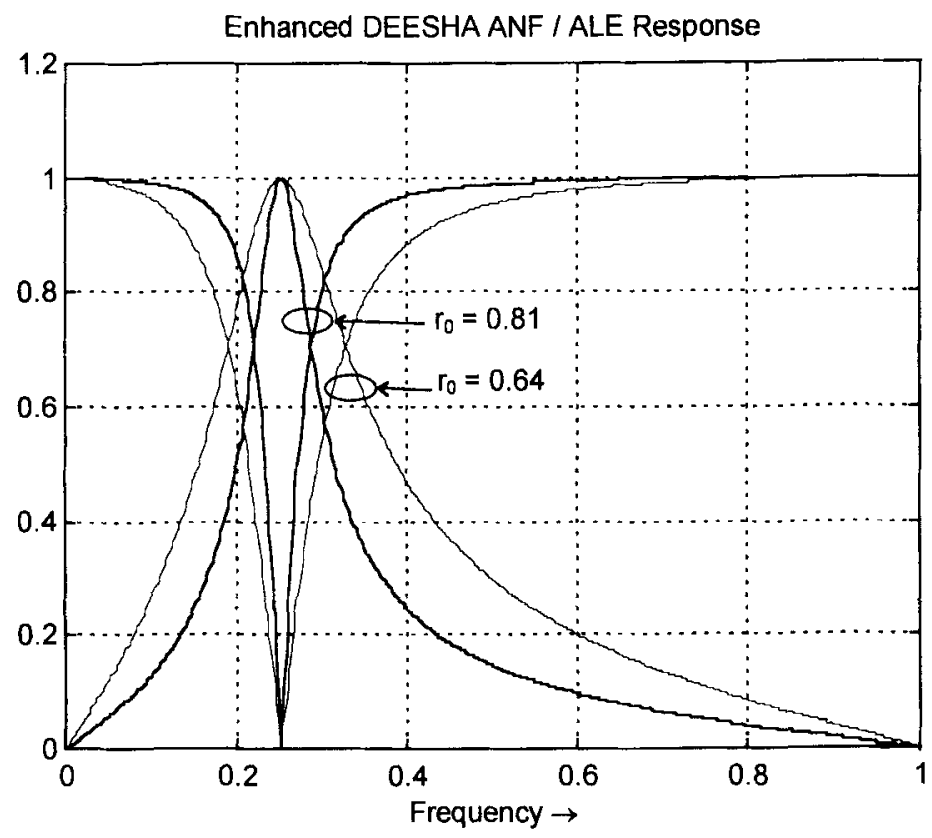

Figure 7. Magnitude response of enhanced DEESHA ANF/ALE. The notch is at $f_{N}=$ 0.25 , and $r_{0}=0.81$ and 0.64 . These characteristics may be compared with those in figure 4 .

derived:

$$
H_{F, 6}(z)=\frac{c_{2}}{2} \frac{\left(1-z^{-2}\right)}{\left[1-\left(2-c_{2}-c_{1}^{2}\right) z^{-1}+\left(1-c_{2}\right) z^{-2}\right]} .
$$

Here, $c_{1}$ tracks the frequency of input sinusoid and has to be adapted, while $c_{2}$ controls the filter bandwidth. A simple eyeballing of (10) and (11) suffices to bring out the relations $r_{0}=1-c_{2}$ and $r_{0}=\left(2-c_{2}-c_{1}^{2}\right) /\left(2-c_{2}\right)$.

The other filter we consider here was proposed by Regalia (1990). Looking once again at only its BPF, we have

$$
H_{F, 7}(z)=\frac{1-\sin \left(\theta_{2}\right)}{2} \frac{\left(1-z^{-2}\right)}{1-\sin \left(\theta_{1}\right)\left[1+\sin \left(\theta_{2}\right)\right] z^{-1}+\sin \left(\theta_{2}\right) z^{-2}},
$$

with obvious relations to enhanced DEESHA given by $\rho_{0}=\sin \left(\theta_{1}\right)$ and $r_{0}=\sin \left(\theta_{2}\right)$. $\theta_{1}$ controls the resonant frequency of ALE (same as notch frequency $\omega_{N}$ of ANF) and can be adapted using any appropriate algorithm to acquire and track the input sinusoid. $\theta_{2}$ determines the filter bandwidth similar to the factor, $r_{0}$ in case of enhanced DEESHA filter.

\section{Conclusions}

In this correspondence, we have established some relationships between different ANF/ ALE designs. Looking back, while some of these inter-relationships seem obvious (in particular, between DEESHA and the "lattice filter" of Cho, Choi and Lee), surprisingly this 
aspect has not been highlighted elsewhere. Even in certain instances where two different notch filters have been examined, invariably the comparison is between a so called "lattice filter" and an inherently biased design such as that of Nehorai's (Cho \& Lee 1993; Regalia 1995).

Our curiosity to explore these relations was aroused by the pole-zero plots of these various designs. We were assisted in this exploration by Farhang-Boroujeny's (1994) recasting of the DEESHA filter function. As the links between the DEESHA filter and others became clear, this naturally led to the simple modification of figure 6 which improves its performance both in notch filtering and in line enhancement.

What is the implication of these relations that have been shown between various biquads for ANF/ALE applications? Mainly we have the assurance that equivalent designs will offer similar asymptotic performance, in terms of bias-free convergence or for ALE, the SNR improvement factor. Also, we hope that the confusion compounded by the wide variety of design choices is reduced by the equivalences discussed here. However, the convergence and tracking behaviour can significantly differ depending on the adaptation technique employed and on the specific parameter being updated. For example, Regalia's design given by Mukund \& Martin (1993) where $\theta_{1}$ is adapted, is quite suited to handle very low or very high frequencies. As for the enhanced DEESHA biquad, either $\rho_{0}$ or $\omega_{N}$ can be adapted. Its acquisition and tracking behaviour for very low or high frequencies will be superior for the latter choice (Farhang-Boroujeny 1994; Krishna \& Hiremath 1995) and will be on par with that of Regalia's. All these issues require careful consideration while selecting a particular biquad design and adaptation approach for a given application.

\section{References}

Cho N I, Lee S U 1993 On adaptive lattice notch filter for the detection of sinusoids. IEEE Trans. Circuits Syst. 40: 405-415

Cho N I, Choi C-H, Lee S U 1990 Adaptive line enhancer using IIR notch filter. IEEE Trans. Acoust. Speech Signal Process. 37: 585-589

Cupo R L, Gitlin R D 1989 Adaptive carrier recovery systems for digital data communications receivers. IEEE J. Selected Areas Commun. JSAC-7: 1328-1339

David R A, Stearns S D, Elliot G R, Etter D M 1983 IIR algorithm for adaptive line enhancement. Proc. IEEE Int. Conf. Acoustics Speech Signal Processing pp 17-20

Farhang-Boroujeny B 1994 An IIR adaptive line enhancer with controlled bandwidth. Proc. Singapore Int. Conf. Circuits and Systems '94 pp 835-839

Hush D R, Ahmed N, David R, Stearns S D 1986 An adaptive IIR structure for sinusoidal enhancement, frequency estimation and detection. IEEE Trans. Acoust. Speech Signal Process. 34: $1380-1389$

Krishna V V, Hiremath C G 1995 Adaptive IIR Notch Filters. Technical Report No. 95-4/BRC, Signion Systems Pvt. Ltd.

Kwan T, Martin K 1989 Adaptive detection and enhancement of multiple sinusoids using a cascade IIR filter. IEEE Trans. Circuits Syst. 36: 937-947

Mukund P, Martin K 1991 Resonator based filter-banks for frequency-domain applications. IEEE Trans. Circuits Syst. 38: 1145-1159

Mukund P, Martin K 1993 A second order hyperstable adaptive filter with no post-error filtering. Proc. IEEE Int. Symp. Circuits and Systems pp 447-450 
Nehorai A 1985 A minimal parameter adaptive notch filter with constrained poles and zeros. IEEE Trans. Acoust. Speech Signal Process. 33: 983-996

Rao D V B, Kung S Y 1984 Adaptive notch filtering for the removal of sinusoids in noise. IEEE Trans. Acoust. Speech Signal Process. 32: 791-802

Regalia P A 1990 A novel lattice based adaptive IIR notch filter. In Signal Processing V: Theories and Applications (eds.) L Torres, E Masgrau, M A Lagunas (Amsterdam: Elsevier) pp 261-264 Regalia P A 1995 Adaptive IIR filtering in signal processing and control (New York: Marcel Dekker) 